\title{
Função pulmonar pós-colecistectomia laparoscópica e abreviado tempo anestésico-cirúrgico
}

\author{
Pulmonary function after laparoscopic cholecystectomy and abbreviated \\ anesthetic-surgical time
}

\author{
Gilson Cassem Ramos; Edísio Pereira 2; Salustiano Gabriel Neto - TCBC-GO3; Enio Chaves de Oliveira- TCBC-GO4
}

R E S U M O

\begin{abstract}
Objetivo: Avaliar a função pulmonar pós-colecistectomias laparoscópicas. Métodos: Estudo prospectivo, onde se avaliaram espirometrias pós-operatórias de 15 pacientes submetidas à colecistectomias laparoscópicas por meio de um tempo anestésicocirúrgico abreviado. Os dados pós-operatórios foram comparados aos pré-operatórios Resultados: Existiram diferenças significativas para as variáveis Capacidade Vital Forçada $(p=0,020)$ e Volume Expiratório Forçado no $1^{\circ}$ segundo $(p=0,022)$ no pré e pósoperatório imediato, indicando distúrbios ventilatórios restritivos. Conclusão: Foram observados distúrbios ventilatórios restritivos leves pós-colecistectomias laparoscópicas, com rápida recuperação da função pulmonar, o que pode diminuir a morbidade pulmonar pós-operatória.
\end{abstract}

Descritores: Pulmão/fisiologia. Testes de função respiratória. Colecistectomia laparoscópica.

\section{INTRODUÇÃO}

O perações realizadas no andar superior do abdome podem induzir a distúrbios ventilatórios restritivos pósoperatórios. Eles são mais acentuados nos procedimentos abertos, contudo são observados também nos laparoscópicos'. Mesmo por meio dessa via, esses procedimentos, em menor monta, promovem inibição reflexa do nervo frênico e disfunção diafragmática ${ }^{2,3}$. No passado, as colecistectomias por via laparoscópica promoviam reduções importantes da Capacidade Vital Forçada (CVF) e do Volume Expiratório Forçado do $1^{\circ}$ segundo $\left(V_{E F}\right)$, semelhantes às observadas pela via aberta ${ }^{4}$. Contudo esperava-se recuperação mais rápida da função pulmonar pós-operatória. Com o domínio da técnica laparoscópica, sabe-se hoje que, indiscutivelmente, é a de primeira escolha para colecistectomias. Não sendo, porém, isenta de enfraquecimento da função pulmonar pós-operatória.

O objetivo deste estudo foi detectar distúrbios ventilatórios restritivos, sua gravidade e o tempo de recuperação das espirometrias nos pós-operatórios de colecistectomias laparoscópicas, relacionadas aos valores pré-operatórios.

\section{MÉTODOS}

Este trabalho foi realizado em pacientes que procuraram o Serviço de Cirurgia do Aparelho Digestivo do
Hospital Ortopédico de Goiânia, Goiânia, Brasil. Ele foi aprovado pelo Comitê de Ética em Pesquisa do Hospital de Urgências de Goiânia e tendo sido obtido o Termo de Consentimento Livre e Esclarecido das enfermas envolvidas na pesquisa.

Foram escolhidos, aleatoriamente, 15 mulheres com idade entre 21 e 65 anos, com IMC menor ou igual a 35, estado físico ASA (American Society of Anesthesiologists) I ou II, com espirometria, gasometria arterial e radiografia de tórax pré-operatórias normais, e que se submeteriam à colecistectomia sem colangiografia intraoperatória. Excluíram-se enfermas em uso de fármacos com efeito broncodilatador; tabagistas; pacientes cuja estatura não pôde ser determinada com precisão (cifoescoliose, amputação de membros inferiores, acamados); gestantes; portadoras de doenças respiratórias; abdome agudo ou com história médica pregressa de doença diverticular do cólon; enfermas com antecedentes de úlcera gastroduodenal; pacientes com antecedentes clínicos de hemorragia digestiva; portadoras de doença neuromuscular; pacientes psiquiátricas; enfermas com contraindicação de receberem bloqueio anestésico peridural; e as com antecedentes clínicos de alergia à dipirona, diclofenaco de sódio ou aos anestésicos selecionados para uso.

É estudo prospectivo, em que as pacientes foram acompanhadas pós-operatoriamente avaliando suas funções pulmonares. As enfermas foram submetidas à colecistectomias por via laparoscópica, sob bloqueio anestésico peridural mais anestesia geral, com protocolo anes-

Trabalho realizado no Programa de Pós-Graduação em Ciências da Saúde da Universidade de Brasília - UnB, Distrito Federal, BR.

1. Doutor em Medicina pela Universidade de Brasília - UnB - DF- BR; 2. Professor Doutor do Programa de Pós-Graduação da Faculdade de Ciências da Saúde da Universidade de Brasília - UnB - DF-BR; 3. Professor Assistente de Técnica Operatória da Faculdade de Medicina da Universidade Federal do Goiás - UFG - GO-BR; 4. Professor Doutor da Disciplina de Coloproctologia da Faculdade de Medicina da UFG - GO-BR. 
tésico padronizado, inclusive com todos os atos anestésicos realizados sempre pelo mesmo anestesiologista. As enfermas foram submetidas à intubação orotraqueal com colocação de cânula número 7,5 e insuflação do balonete com $5 \mathrm{~mL}$ de ar. A anestesia foi mantida com isofluorano $(0,5 \mathrm{a}$ $1 \%$ ) e $\mathrm{N}_{2} \mathrm{O}$, em uma mistura de $50 \% \mathrm{com} \mathrm{O}_{2}$. Demarcouse o tempo de duração da intubação, do momento em que a extremidade inferior da cânula atravessava as cordas vocais até a ocasião da extubação orotraqueal, procedimento indicado quando a paciente apresentava amplitude e frequência respiratória clinicamente satisfatórias. Os valores, em minutos da intubação foram arredondados para números inteiros, de forma que o valor considerado na pesquisa foi no máximo 30 segundos de diferença do valor real cronometrado. Procedimento cujo tempo anestésicocirúrgico ultrapassava 50 minutos, inclusive com extubação orotraqueal, era excluído do estudo e nova paciente era selecionada para compor o grupo. Dipirona $2 \mathrm{~g}$ endovenosa e diclofenaco de sódio $75 \mathrm{mg}$ intramuscular, foram administrados, respectivamente, de 6 em 6 horas e de 12 em 12 horas, iniciando na alta da sala de recuperação pósanestésica.

As pacientes foram operadas sempre pelo mesmo cirurgião, com a mesma técnica cirúrgica ${ }^{5}$. Eram posicionadas em decúbito dorsal horizontal. Realizava-se uma incisão na borda superior da cicatriz umbilical, interessando pele e subcutâneo. Introduzia-se um trocarte de 10 $\mathrm{mm}$, iniciando a insuflação de $\mathrm{CO}_{2}$. Estabelecido o pneumoperitôneo (mantido com pressão em torno de 13 $\mathrm{mmHg}$ ), introduzia-se a câmara e, sob visão direta, procedia-se a inserção de trocartes adicionais na cavidade peritoneal: um de $10 \mathrm{~mm}$ na região subxifóide e dois de $5 \mathrm{~mm}$ na região subcostal direita, na linha hemiclavicular e na linha axilar anterior. O procedimento era realizado dissecando-se o peritônio do colo da vesícula e o ducto cístico. Após a identificação da artéria e ducto císticos, realizavase a clipagem e a secção dessas estruturas. Fazia-se a remoção da vesícula pela secção do peritônio e da adventícia, entre o fígado e a vesícula. A hemostasia era realizada com uso de eletrocautério. A vesícula era retirada pelo orifício subxifóide e a pele suturada. Delimitou-se o tempo da operação em minutos, servindo do mesmo método de aproximação empregado para se obter o tempo de duração da intubação. O início da operação correspondia ao momento da incisão da pele e, o final, ao último ponto na pele.

As enfermas submeteram-se à espirometrias seriadas. O primeiro exame era realizado no pré-operatório. O segundo, no dia seguinte ao procedimento nas 24 horas do pós-operatório imediato. A partir deste momento, a cada dois dias, realizaram-se novas espirometrias até a obtenção de um teste considerado normal para a paciente analisada, momento em que se interrompia a realização de novos testes. As espirometrias foram realizadas sempre pela mesma profissional - técnica em função pulmonar - e utilizando-se o mesmo aparelho: espirômetro portátil Spiro Pro ${ }^{\circledR}$ versão 2.0, validado pela American Thoracic Society (ATS), capaz de medir parâmetros pulmonares de fluxo e volume. Esse equipamento, além de gerar as curvas de Fluxo/Volu- me e de Volume/Tempo, discriminava 12 variáveis espirométricas e os resultados impressos automaticamente. Os parâmetros analisados foram baseados na equação de regressão de Knudson ${ }^{6}$. A preparação para cada sessão de espirometria incluía calibrar o espirômetro com seringa apropriada, ajustado de acordo com a temperatura ambiente $\left(25 \mathrm{a} 40^{\circ} \mathrm{C}\right)$ e pressão atmosférica $(680 \mathrm{mmHg})$.

Foram proscritos para as pacientes, nas últimas quatro a seis horas, bebida alcoólica, alimentos que contivessem cafeína - tais como chá, café, chocolate e refrigerante tipo coca-cola pelo seu efeito broncodilatador ${ }^{7}$. As variáveis individuais, estatura (em cm), peso (em kg), gênero feminino e data de nascimento, eram coletadas e armazenadas no espirômetro. Após dez minutos de repouso em ambiente calmo, a enferma era orientada a se colocar em posição sentada e a focar sua atenção para a explicação do procedimento, que era descrito, criteriosamente, dando-se ênfase à necessidade de evitar vazamentos em torno da peça bucal descartável e à importância da inspiração máxima seguida de expiração rápida, potente (explosiva) e sustentada, até que se ordenasse sua interrupção. A narina era obstruída com clipe nasal e o teste realizado em um sistema do tipo fechado. Cada paciente submetia-se a três testes válidos e reprodutíveis. O aparelho Spiro Pro ${ }^{\circledR}$ versão 2.0 utiliza o maior valor obtido da equação CVF + $V E F$, para selecionar o melhor teste. Os laudos das espirometrias foram fornecidos sempre pelo mesmo pneumologista, especialista em provas de função pulmonar, que as interpretava sem conhecer a história clínica da paciente. As variáveis CVF e VEF foram analisadas, separadamente, no pré e pós-operatórios, até o momento de suas normalizações (80 \% do valor teórico pré-calculado para $\left(V F\right.$ e $\left.V E F_{1}\right)$. A hipótese de igualdade de médias do grupo, antes e após intervenção cirúrgica, foi verificada por meio do teste t-Student emparelhado ${ }^{8}$. Valor de $p<0,05$ foi considerado estatisticamente significativo na avaliação de diferenças entre parâmetros.

\section{RESULTADOS}

A tabela 1 refere-se às características demográficas das pacientes e as variáveis operatórias.

A tabela 2 refere-se às variáveis CVF e VEF, do pré-operatório ao terceiro dia de pós-operatório. Exis-

Tabela 1 - Características das pacientes estudadas com as variáveis operatórias (média \pm desvio padrão)

\begin{tabular}{lr}
\hline Variáveis & Colecistectomia \\
\hline - Idade (anos) & $44,13 \pm 11,17$ \\
- Altura (cm) & $153,20 \pm 2,23$ \\
- Peso (kg) & $65,33 \pm 9,36$ \\
- IMC & $27,66 \pm 4,10$ \\
- Tempo cirúrgico (min) & $24,60 \pm 6,52$ \\
- Tempo de IOT (min) & $40,80 \pm 6,68$ \\
\hline
\end{tabular}

$I M C=$ peso $(\mathrm{kg}) /$ altura $\left(\mathrm{m}^{2}\right) ; \min =$ minutos; IOT=intubação orotraqueal. 
tiram diferenças estatisticamente significativas para ambas as variáveis, quando se compararam pré $X$ pósoperatório imediato $(p=0,020$ para CVF e $p=0,022$ para $V E F_{1}$ ). A tabela 3 refere-se aos valores individuais de cada uma das variáveis espirométricas, no pré e pósoperatórios

A figura 1 refere-se às reduções das variáveis espirométricas CVF e VEF, no pré-operatório e no pós-operatório imediato. Já a figura 2 ilustra a curva dessas mesmas variáveis espirométricas do pré-operatório e $3^{\circ}$ dia de pós-operatório.

\section{DISCUSSÃO}

No presente estudo observou-se a presença de distúrbios ventilatórios restritivos leves, com redução da CVF e do $V_{E F}$, quando essas duas variáveis foram comparadas pré e pós-operatoriamente. Assim, colecistectomia por via laparoscópica também evolui com alterações espirométricas pós-operatórias, observação que se identifica com várias outras publicações científicas ${ }^{9,10}$. Contudo, nesta pesquisa, a diminuição mais acentuada da CVF e do VEF foi, respectivamente, de $8,2 \%$ e $8,4 \%$ em relação aos valores pré- operatórios. Isso equivale a dizer que os valores espirométricos pós-operatórios são de exames normais, comparados aos valores preditos.

Não foram encontrados relatos de observações científicas equivalentes aos verificados nesse estudo. Vêmse, habitualmente, alterações mais pronunciadas, mesmo nas laparoscopias, com reduções entre $20 \%$ e $30 \%{ }^{11-13} \mathrm{em}$ ambas as variáveis ou até mesmo quedas mais significativas de mais de $40 \%{ }^{4}$.

A disfunção diafragmática é o fator causal mais importante relacionado aos distúrbios ventilatórios restritivos pós-colecistectomias ${ }^{2,14,15}$ e ela está presente, inclusive, nos procedimentos por laparoscopia ${ }^{1,16}$. Essa disfunção independe da dor pós-operatória², dura cerca de uma semana, é mediado por mecanismo reflexo aferente de inibição do nervo frênico ${ }^{15}$, e o diafragma não se apresenta com contratilidade prejudicada ${ }^{17}$. Outro fator importante na gênese dos distúrbios ventilatórios é a dor pós-operatória, que também contribui para a deterioração da função pulmonar após procedimentos cirúrgicos de abdome superior ${ }^{18}$.

Outros fatores também são relevantes, pois aumentam a disfunção diafragmática e a dor pós-operatória e tendem a enfraquecer a função pulmonar, dentre os quais

Tabela 2 - Variáveis espirométricas: comparação do pré X pós-operatório imediato e pré X $3^{\circ}$ dia pós-operatório (PO).

\begin{tabular}{|c|c|c|c|c|c|c|c|c|c|c|}
\hline \multirow[t]{3}{*}{ Variáveis } & \multicolumn{6}{|c|}{ Comparação } & \multicolumn{4}{|c|}{ Estatística } \\
\hline & \multicolumn{2}{|c|}{ Pré-operatório } & \multirow{2}{*}{$\begin{array}{l}1^{\circ} \mathrm{PO} \\
\text { Média }\end{array}$} & \multicolumn{3}{|c|}{$3^{\circ} \mathrm{PO}$} & \multicolumn{2}{|c|}{ Pré X PO } & \multicolumn{2}{|c|}{ Pré X PO } \\
\hline & Média & $\begin{array}{l}\text { Desvio } \\
\text { Padrão }\end{array}$ & & $\begin{array}{l}\text { Desvio } \\
\text { Padrão }\end{array}$ & Média & $\begin{array}{l}\text { Desvio } \\
\text { Padrão }\end{array}$ & $\mathrm{t}$ & $p$ & $\mathrm{t}$ & $p$ \\
\hline CVF & 3,30 & 0,32 & 3,03 & 0,42 & 3,33 & 0,28 & 2,628 & 0,020 & $-0,409$ & 0,689 \\
\hline $\mathrm{VEF}_{1}$ & 2,73 & 0,33 & 2,50 & 0,38 & 2,89 & 0,26 & 2,567 & 0,022 & $-2,161$ & 0,049 \\
\hline
\end{tabular}

Tabela 3 - Valores individuais (litros) das variáveis espirométricas.

\begin{tabular}{|c|c|c|c|c|c|c|}
\hline \multirow[b]{2}{*}{ Ordem } & \multicolumn{2}{|c|}{ Pré-operatório } & \multicolumn{2}{|c|}{ PO imediato } & \multicolumn{2}{|c|}{$3^{\circ} \mathrm{PO}$} \\
\hline & CVF & $\mathrm{VEF}_{1}$ & CVF & VEF $_{1}$ & CVF & $\mathrm{VEF}_{1}$ \\
\hline 1 & 2,82 & 2,40 & 3,73 & 3,12 & 3,80 & 3,34 \\
\hline 2 & 3,64 & 3,08 & 3,43 & 2,96 & 3,57 & 3,03 \\
\hline 3 & 3,61 & 2,88 & 3,35 & 2,72 & 3,60 & 3,20 \\
\hline 4 & 3,44 & 2,84 & 2,86 & 2,40 & 3,48 & 3,13 \\
\hline 5 & 3,55 & 2,92 & 2,77 & 2,36 & 3,55 & 2,94 \\
\hline 6 & 3,23 & 2,84 & 2,99 & 2,32 & 3,27 & 2,87 \\
\hline 7 & 2,92 & 2,48 & 2,32 & 1,96 & 3,10 & 3,00 \\
\hline 8 & 3,95 & 3,52 & 3,85 & 3,32 & 3,85 & 3,27 \\
\hline 9 & 3,34 & 2,84 & 2,79 & 2,32 & 3,20 & 2,72 \\
\hline 10 & 2,99 & 2,52 & 2,54 & 2,12 & 3,10 & 2,70 \\
\hline 11 & 3,23 & 2,24 & 2,96 & 2,32 & 3,23 & 2,61 \\
\hline 12 & 3,10 & 2,48 & 3,05 & 2,48 & 3,05 & 2,57 \\
\hline 13 & 3,54 & 2,88 & 3,24 & 2,60 & 3,24 & 2,88 \\
\hline 14 & 3,29 & 2,68 & 2,65 & 2,08 & 3,05 & 2,68 \\
\hline 15 & 2,92 & 2,40 & 2,94 & 2,44 & 2,94 & 2,52 \\
\hline
\end{tabular}

PO = Pós-operatório 


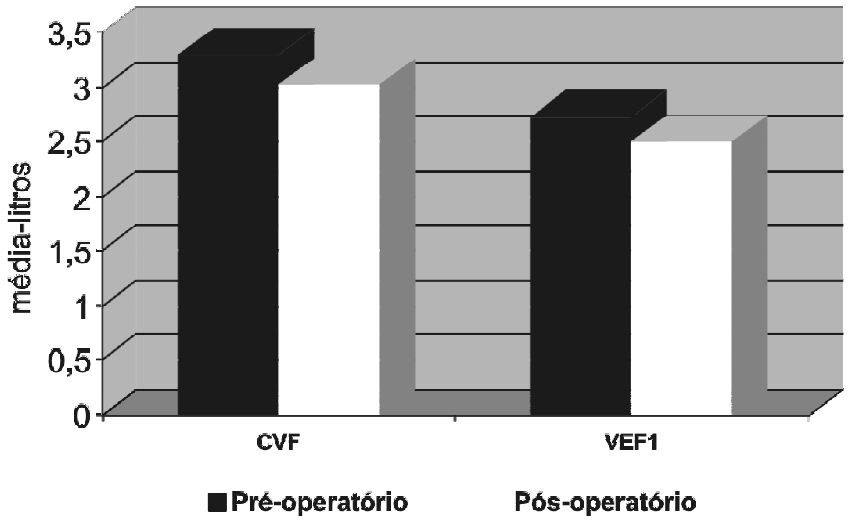

Figura 1 - CVF e VEF : pré-operatório e pós-operatório imediato.

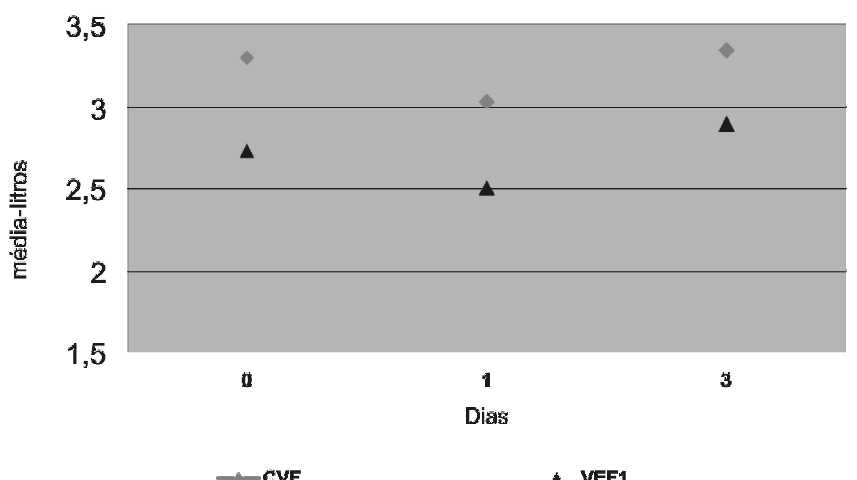

Figura 2 - CVF e VEF do período pré-operatório até o $3^{\circ}$ dia de pós-operatório. a duração da agressão tecidual (tempo cirúrgico prolongado acima de uma ou duas horas), o tamanho da incisão cirúrgica, a lesão de fibras musculares e tempo de intubação maior que duas horas ${ }^{19,20}$.

Assim, neste experimento, menor disfunção diafragmática e dor pós-operatória, próprias dos procedimentos laparoscópicos ${ }^{21,22}$, associadas a tempo anestésico-cirúrgico rápido, foram os principais responsáveis por espirometrias pós-operatórias minimamente alteradas ou normais, relacionadas aos valores preditos. Todas as pacientes apresentaram, no pósoperatório imediato, espirometrias consideradas normais, porém inferiores aos valores dno período préoperatório. Assim, as variáveis espirométricas CVF e VEF, diminuíram dos valores pré em relação aos do pós-operatório imediato, e na medida seguinte $\left(3^{\circ}\right.$ dia de pós-operatório), os valores já se equivaleram aos do pré-operatório. Algumas pesquisas apontaram para recuperação da função pulmonar pós-colecistectomia laparoscópica entre oito a dez dias ${ }^{18,23}$, o que não encontra amparo no presente estudo. Provavelmente, essa diferença poderia ser justificada pelo tempo cirúrgico abreviado, com uma injúria tecidual e disfunção diafragmática menores.

Assim, pôde-se concluir que a função pulmonar após colecistectomia laparoscópica com tempo anestésico-cirúrgico abreviado, tende a recuperação mais rápida, o que pode diminuir a morbidade pulmonar pósoperatória.

\section{A $B$ S T $R$ R A C T}

Objective: To evaluate pulmonary function after laparoscopic cholecystectomies. Methods: Prospective study, in which the postoperative spirometries of 15 patients who underwent laparoscopic cholecystectomies with abbreviated anesthetic-surgical time were analyzed. Results: Significant differences existed for the Forced Vital Capacity variable $(p=0,020)$ and Forced Expiratory Volume in the first second $(p=0,022)$ between pre- and immediate post-operative, indicating restrictive ventilatory disturbances. Conclusion: Light restrictive laparoscopic post-cholecystectomy ventilatory disturbances were observed, with rapid recovery of pulmonary function, which may lower post-operative pulmonary morbidity.

Key words: Lung/physiology. Respiratory function tests. Cholecystectomy, laparoscopic.

\section{REFERÊNCIAS}

1. Erice F, Fox GS, Salib YM, Romano E, Meakins JL, Magder SA. Diaphragmatic function before and after laparoscopic cholecystectomy. Anesthesiology. 1993; 79(5):966-75; discussion 27A-28A.

2. Simonneau G, Vivien A, Sartene R, Kunstlinger F, Samii K, Noviant $Y$, Duroux P. Diaphragm dysfunction induced by upper abdominal surgery. Role of postoperative pain. Am Rev Respir Dis. 1983; 128(5):899-903.

3. Ford GT, Whitelaw WA, Rosenal TW, Cruse PJ, Guenter CA. Diaphragm function after upper abdominal surgery in humans. Am Rev Respir Dis. 1983; 127(4):431-6.

4. Barnett RB, Clement GS, Drizin GS, Josselson AS, Prince DS. Pulmonary changes after laparoscopic cholecystectomy. Surg Laparosc Endosc. 1992; 2(2):125-7.

5. Smadja C, Blumgart L. The biliary tract and the anatomy of biliary exposure. In: Blumgart L. Surgery of the liver and biliary tract. New York: Churcill Livingstone; 1994. p. 11-24.
6. Knudson RJ, Lebowitz MD, Holberg CJ, Burrows B. Changes in the normal maximal expiratory flow-volume curve with growth and aging. Am Rev Respir Dis. 1983; 127(6):725-34

7. Sociedade Brasileira de Pneumologia e Tisiologia. I Consenso brasileiro sobre espirometria. J Pneumol. 1996; 22(3):122-9.

8. Vieira S. Teste t. In: Vieira S. Introdução a bioestatística. $3^{\text {a }}$ ed. Rio de Janeiro: Campus; 1980. p. 121-36.

9. Ramos GC, Pereira E, Gabriel Neto S, Oliveira EC, Rassi RH, Lemos Neto SP. Influência da morfina peridural na função pulmonar de pacientes submetidos à colecistectomia aberta. Rev Bras Anestesiol. 2007; 57(4):366-81.

10. Mahul P, Burgard G, Costes F, Guillot B, Massardier N, El Khouri Z et al. Postoperative respiratory function and cholecystectomy by laparoscopic approach. Ann Fr Anesth Reanim. 1993; 12(3):273-7.

11. Frazee RC, Roberts JW, Okeson GC, Symmonds RE, Snyder SK Hendricks JC, Smith RW. Open versus laparoscopic cholecystectomy. A comparison of postoperative pulmonary function. Ann Surg. 1991; 213(6):651-3. 
12. Hasukiæ S, Mesiæ D. Postoperative pulmonary changes after laparoscopic cholecystectomy. Med Arh. 2001; 55(2):91-3.

13. Ravimohan SM, Kaman $L$, Jindal R, Singh R, Jindal SK. Postoperative pulmonary function in laparoscopic versus open cholecystectomy: prospective, comparative study. Indian J Gastroenterol. 2005; 24(1):6-8.

14. Dureuil B, Cantineau JP, Desmonts JM. Effects of upper or lower abdominal surgery on diaphragmatic function. Br J Anaesth. 1987; 59(10): 1230-5.

15. Sprung J, Cheng EY, Nimphius N, Hubmayr RD, Rodarte JR, Kampine JP. Diaphragm dysfunction and respiratory insufficiency after upper abdominal surgery. Plucne Bolesti. 1991; 43(1-2):5-12.

16. Joris J, Kaba A, Lamy M. Postoperative spirometry after laparoscopy for lower abdominal or upper abdominal surgical procedures. $\mathrm{Br} \mathrm{J}$ Anaesth. 1997; 79(4):422-6.

17. Dureuil B, Viires N, Cantineau JP, Aubier M, Desmonts JM. Diaphragmatic contractility after upper abdominal surgery. J Appl Physiol. 1986; 61(5):1775-80.

18. de La Peña M, Togores B, Bosch M, Maimo A, Abad S, Garrido P et al. Recuperación de la función pulmonar tras colecistectomía laparoscópica: papel del dolor postoperatorio. Arch Bronconeumol. 2002; 38(2):72-6.

19. Kroenke K, Lawrence VA, Theroux JF, Tuley MR. Operative risk in patients with severe obstructive pulmonary disease. Arch Intern Med. 1992; 152(5):967-71.

20. Wong DH, Weber EC, Schell MJ, Wong AB, Anderson CT, Barker SJ. Factors associated with postoperative pulmonary complications in patients with severe chronic obstructive pulmonary disease. Anesth Analg. 1995; 80(2):276-84

21. Schauer PR, Luna J, Ghiatas AA, Glen ME, Warren JM, Sirinek KR. Pulmonary function after laparoscopic cholecystectomy. Surgery. 1993; 114(2):389-97.

22. Ellstrom M, Olsen MF, Olsson JH, Nordberg G, Bengtsson A, Hahlin $M$. Pain and pulmonary function following laparoscopic and abdominal hysterectomy: a randomized study. Acta Obstet Gynecol Scand. 1998; 77(9):923-8.

23. Bablekos GD, Roussou T, Rasmussen T, Vassiliou MP, Behrakis PK. Postoperative changes on pulmonary function after laparoscopic and open cholecystectomy. Hepatogastroenterology. 2003; 50(53):1193- 1200

Recebido em 14/11/2008

Aceito para publicação em 19/01/2009

Conflito de interesse: nenhum

Fonte de financiamento: nenhuma

Como citar este artigo:

Ramos GC, Pereira E, Gabriel Neto S, Oliveira EC. Função pulmonar pós-colecistectomia laparoscópica e abreviado tempo anestésico-cirúrgico. Rev Col Bras Cir. [periódico na Internet] 2009; 36(4). Disponível em URL: http://www.scielo.br/rcbc

Endereço para correspondência:

Gilson Cassem Ramos

E-mail: gilson.ramos@terra.com.br 